\title{
HUGO Statement on Pharmacogenomics (PGx): Solidarity, Equity and Governance
}

\section{HUMAN GENOME ORGANISATION ETHICS COMMITTEE}

\section{Reason for Statement}

The HUGO Ethics Committee,

- Recognising that there have been significant discussions of the ethical issues arising in the application of genetic knowledge; ${ }^{1}$

- Recognising that PGx has the potential to maximize therapeutic outcomes and minimize adverse reactions to therapy, and that it is consistent with the traditional goals of public health and medical care to relieve human suffering and save lives;

- Recognising that the ethical, research and policy issues that need to be considered in PGx include:

○ the ways in which PGx requires a novel focus on families, populations and communities;

○ the impact of 'personalised' medicine on a society;

o the implications for populations in developing countries, including access to therapies for neglected diseases;

o the impact of PGx on health care costs and policies worldwide;

O the significance of PGx for research priorities;

o the importance of re-evaluation of prior clinical trial data for efficacy among particular genotypes (as in resuscitation of abandoned drugs);

o the significance of PGx for existing, as opposed to new, drugs; and

0 the fear that PGx could reinforce genetic determinism and lead to discrimination against, and stigmatization of, individuals and groups;

hereby identifies a pressing need to reach consensus on the most important ethical principles that are applicable and for workable guidelines in clinical and public health settings.

\section{Definitions and scope}

There has been extensive discussion of the relative benefits of using the terms pharmacogenomics (PGx) and pharmacogenetics, and various definitions have been suggested. To some extent the terms have been used interchangeably. Although both 'genetics' and 'genomics' are popularly used in different ways in different cultures, and have different meanings in a variety of languages, in this statement we use the term pharmacogenomics (PGx) and understand 'PGx' to mean the total sum of genetic variation that affects response to therapeutic agents.

The Committee recognises that principles developed in this Statement on PGx also apply to other therapeutic modalities such as ionising radiation and biologicals, and to variation in response in contexts such as nutrition, environmental exposures and toxicology. 


\section{Principles}

Past HUGO Ethics Committee Statements have reflected a commitment to the view that the highest ethical priority in implementing genomic knowledge is that of saving life and reducing suffering, but the Committee considers it urgent that the ethical principles of solidarity and equity be given increased attention

- Solidarity: Because of shared vulnerabilities, people have common interests and moral responsibilities to each other. Willingness to share information and to participate in research is a praiseworthy contribution to society.

- Equity: To reduce health inequalities between different populations, and to work towards equal access to care is an important prerequisite for implementing genomic knowledge for the benefit of society.

while also reaffirming the following long-accepted ethical considerations:

- Respect for human rights

- Protection of confidentiality and privacy

- Avoiding harm

- Beneficence: there is an obligation to do good, and to maximise the possible benefits of genomics, which should be regarded as a global public good

- Autonomy: the freedom of persons to make decisions regarding their medical care is fundamental to the modern practice of medicine

- The authority of communities to participate in decisions that affect them

- The fundamental relationship between the quality of scientific research and its ethical acceptability

\section{Recommendations}

\section{Research priorities}

1.1. There needs to be a careful consideration of research priorities in PGx and translational research for each society and these should not merely be led by economic priorities determined by market forces.

1.2. The continuing creation of a sound scientific basis for PGx should be pursued, such as:

identification of genetic factors, including genes, haplotypes, SNPs and copy number variants (CNVs) with significant pharmacogenomic effects; identification of interactions with other genetic, environmental and social factors among drugs currently in common use in different countries; metaanalyses for consistency of data; ongoing development of methods for biomarker analyses that are suitable for large scale studies.

1.3. The continuing creation of a sound corpus of research on ethical and legal issues, appropriate governance, social science and policy research, and methods of community and public dialogue and participation. 


\section{Governance of research}

2.1. There needs to be appropriate governance at national and international levels of the collection, storage, utilization, sharing, and protection of data and biological specimens.

2.2. There is an urgent need for institutions and scientists to apply the principle of open access and sharing of data, consistent with the protection of personal privacy of the persons contributing to the database, in order to maximise benefit.

2.3. The establishment of necessary infrastructure, including international initiatives for sharing data between biobanks, should be supported.

2.4. There should be support and encouragement by research funding agencies to require and support the sharing of data.

2.5. HUGO should consider establishing mechanisms for on-going international co-ordination and evaluation of developments in PGx, with special reference to harmonising standards for reliability and replicability of PGx association studies.

2.6. While many institutions have developed governance mechanisms, such as ethics committees, attention should be paid to preparing them to assess appropriately the benefits and risks of PGx protocols.

\section{Maximising the benefits of research}

3.1. It should be recognised that PGx can be of benefit to communities as well as to individuals, even in the absence of optimal infrastructure/resources

3.2. In order to reduce health inequalities, there is a need both to develop new drugs for people with certain genetic variants, especially in the case of neglected and orphan diseases, and to consider the possibility of resuscitation of abandoned drugs for particular population groups.

\section{Participation in research and social responsibility}

4.1. All stakeholders in PGx research should exercise their ethical responsibilities in a spirit of equity and solidarity.

4.2. Voluntary participation of members of a community in PGx research provides an opportunity to actualise the principle of solidarity. Researchers have an obligation to engage the community while maintaining the highest standard of research conduct to earn the trust of the community.

4.3. The participation of all stakeholders, including the wider community, in such research requires public and professional dialogue and education in the science and ethics of PGx.

\section{Clinical implementation}

5.1. The principle of equity implies that therapies should be made equally available to those with equal needs. If this were not the case, the translation of genomic knowledge into clinical practice would aggravate disparities among people. 
5.2. Barriers to translation of genomic knowledge into practice should be identified and addressed, e.g., by training of appropriate personnel; by working towards more equitable health care systems; etc.

5.3. An individual's serious side effect, or absence of response, may have important implications for drug treatment of blood relatives. The unit of care with respect to drug treatment may include the family as well as the individual, e.g., physicians should be alert to the implications for the relatives of a patient who suffers a serious adverse drug reaction, and should initiate genetic counselling.

5.4. Voluntary sharing of PGx information within families should be encouraged.

\section{Monitoring and quality control}

6.1. There needs to be agreement upon a standard set of data to be collected and a common format to facilitate international data sharing.

6.2. Systematic recording of clinical drug reactions to obtain data useful for PGx research should be encouraged.

6.3. Robust mechanisms of quality control need to be in place to minimise the possibility of error in individual PGx testing and maintenance of patient records.

6.4. Regulatory agencies should address the implications of PGx practice on a wide scale, including the possibilities of off-label use and liability issues.

\section{Education, training and awareness}

7.1. In the light of the rapid growth in PGx knowledge there is an urgent need to increase the level of awareness, education and training in the above issues for all stakeholders, including researchers, clinicians, policy makers, social scientists, patients and publics.

\section{The HUGO Ethics Committee}

Professor Kåre Berg (Vice Chair), Norway; Professor Ruth F. Chadwick (Chair), UK; Professor Jose Maria Cantu, Mexico; Professor Abdallah S. Daar, Oman and Canada; Professor Kazuto Kato, Japan; Professor Darryl R. J. Macer, New Zealand and Thailand; Professor John J. Mulvihill, USA; Professor Thomas H. Murray, USA; Professor Carlos M. Romeo-Casabona, Spain; Professor Ishwar Verma (Vice Chair), India; Professor Zhai Xiaomei, China

Montreal, May 2007

\footnotetext{
${ }^{1}$ The Universal Declaration on Bioethics and Human Rights was unanimously adopted on 19 October 2005 by all member countries of UNESCO. It follows two specific Declarations on ethics of applications of genomics that all member countries of UNESCO have agreed to; the Universal Declaration on the Human Genome and Human Rights (1997), also adopted unanimously by all UN members in 1998, and the International Declaration on Human Genetic Data (2003). In addition there have been many declarations and scholarly articles, and proclamations by agencies and committees of national governments, which provide insight into the theoretical issues and practical applications of ethics to issues raised by genomics, that we draw upon herein.
} 